\title{
Incidência e Preditores de Revascularização da Lesão-Alvo no Seguimento Clínico de Longo Prazo: Análise Crítica do Registro DESIRE
}

\author{
Luciano Pessoa Cavalcante', Amanda G. M. R. Sousa', Ricardo A. Costa' ${ }^{1}$, Adriana Moreira', \\ José Ribamar Costa Jr. ${ }^{1}$, Galo Maldonado', Manuel Cano', Cantídio Campos ${ }^{1}$, Mariana Carballo', \\ Otávio Berwanger ${ }^{1}$, Adib D. Jatene ${ }^{1}$, J. Eduardo Sousa ${ }^{1}$
}

\section{RESUMO}

Introdução: Reportamos a incidência e preditores de nova revascularização da lesão-alvo (RLA) numa coorte de pacientes do mundo real tratados com stents farmacológicos (SF). Método: Entre maio de 2002 e fevereiro de 2010, 3.320 pacientes de único centro foram submetidos a intervenção percutânea (ICP), eletiva ou de urgência, com implante de $5.320 \mathrm{SF}$ (Cypher, 83\%; Taxus, 9\%; Xience V/Promus, 3\%; Endeavor, $4 \%$; BioMatrix, $1 \%)$. O seguimento clínico de até oito anos (mediana, 3,4 anos) foi completo em 98\% do grupo e 129 pacientes $(3,88 \%)$ foram submetidos a RLA. Resultados: Entre os pacientes com RLA, 33\% tinham diabetes, 10\% eram portadores de insuficiência renal e $61 \%$, de doença em múltiplos vasos. A artéria descendente anterior foi o vaso mais frequentemente tratado $(33 \%)$, e $72,3 \%$ das lesões eram de alta complexidade (tipo B2/C). Nesse grupo, foram implantados 214 SF em 191 lesões e 45\% dos pacientes receberam múltiplos stents. $\mathrm{Na}$ análise univariada, os preditores de RLA foram: diabetes melito [razão de risco (RR) 1,88, intervalo de confiança (IC) de 95\% 1,08-3,27; $P=0,025)$, uso do stent Taxus (RR 1,73, IC 95\% 1,20-2,50; P = 0,003), lesão ostial (RR 1,82, IC 95\% $1,1-2,98 ; P=0,018$ ), ponte de safena (RR 2,36; IC 95\% 1,55-3,50; $p=0,0001$ ), e lesões reestenóticas (RR 1,95, IC 95\% 1,17-3,24; $P=0,009)$. Após análise multivariada, permaneceram como preditores independentes: diabetes melito, uso do stent Taxus e tratamento de lesões em pontes de safena (RR 1,64, IC 95\% 1,14-2,35; P = 0,008). Conclusão: No registro DESIRE, a RLA em pacientes complexos tratados com SF foi baixa, e o diabetes melito,

\section{ABSTRACT}

Incidence and Predictors of Target Lesion Revascularization During the Long-term Clinical Follow-up: A Critical Analysis of the DESIRE Registry

Background: We report the incidence and predictors of target lesion revascularization (TLR) in a cohort of realworld patients treated with drug eluting stents (DES). Method: From May 2002 to February 2010, 3,320 patients were treated with elective or emergency percutaneous coronary intervention ( $\mathrm{PCl}$ ) and 5,320 DES (Cypher, 83\%; Taxus, 9\%; Xience V/Promus, 3\%; Endeavor, 4\%; BioMatrix, 1\%) were implanted in a single center. The clinical follow-up of up to eight years (median time 3.4 years) was concluded in $98 \%$ patients of the group and 129 patients (3.88\%) were submitted to TLR. Results: Of the patients with TLR, 33\% had diabetes, $10 \%$ had chronic kidney disease and $61 \%$ had multivessel disease. The left anterior descending artery was the most frequent target vessel (33\%) and $72.3 \%$ of the lesions were complex lesions (type B2/C). In this group, 214 DES were implanted in 191 lesions and $45 \%$ of the patients received multiple stents. In the univariate analysis, TLR predictors were: diabetes mellitus [relative risk (RR) $1.88,95 \%$ confidence interval $(\mathrm{Cl}) 1.08-3.27 ; \mathrm{P}=0.025]$, use of Taxus stents (RR 1.73, 95\% Cl 1.20-2.50; $\mathrm{P}=0.003$ ), ostial lesion ( $R R 1.82,95 \% \mathrm{Cl} 1.1-2.98 ; \mathrm{P}=0.018$ ), saphenous bypass graft (RR 2.36, 95\% Cl 1.55-3.50; $\mathrm{P}=0.0001)$, and restenotic lesions ( $R R$ 1.95, 95\% Cl 1.17-3.24; $\mathrm{P}=0.009$ ). After the multivariate analysis, the independent predictors were: diabetes mellitus, use of Taxus stents and treatment

1 Hospital do Coração da Associação do Sanatório Sírio - São Paulo, SP, Brasil.

Correspondência: Luciano Pessoa Cavalcante. Rua Desembargador Eliseu Guilherme, 123/147 - Paraíso - São Paulo, SP, Brasil CEP 04004-030

E-mail: pessoa.Iuciano@gmail.com

Recebido em: $17 / 4 / 2010$ - Aceito em: 31/5/2010 
o tratamento de lesões em ponte de safena e o uso de SF Taxus foram os únicos preditores de RLA no acompanhamento clínico de até oito anos.

DESCRITORES: Stents. Angioplastia. Reestenose coronária.

A introdução de stents farmacológicos na prática clínica revolucionou o tratamento percutâneo da doença coronária. Os excelentes resultados clínicos obtidos com o uso de stents farmacológicos nos mais diversos cenários da prática intervencionista fizeram com que esses novos dispositivos ocupassem papel central no tratamento percutâneo da doença aterosclerótica coronária.

Vários estudos randomizados comparando stents farmacológicos e stents não-farmacológicos demonstraram benefício significativo dos stents farmacológicos, principalmente pela marcante redução da necessidade de revascularização da lesão-alvo. ${ }^{1-4}$ É importante notar que não se evidenciou diferença significativa entre stents farmacológicos e stents não-farmacológicos em relação ao perfil de segurança no acompanhamento clínico tardio. Assim, os stents farmacológicos foram rapidamente incorporados à rotina da intervenção coronária percutânea desde o início de sua comercialização no Brasil (maio de 2002) e sua utilização foi ampliada para pacientes de maior complexidade tanto clínica como angiográfica.

Vários estudos randomizados avaliaram preditores de revascularização da lesão-alvo após o uso de stents farmacológicos. Entretanto, há poucos dados sobre os potenciais preditores desse evento no seguimento de longo prazo de populações não selecionadas. Nosso objetivo foi identificar a incidência e os preditores de revascularização da lesão-alvo na evolução tardia de uma grande coorte de pacientes da prática clínica diária.

\section{MÉTODO}

\section{Protocolo e população estudada}

O Registro DESIRE é um estudo clínico prospectivo, não-randomizado, de braço único, com inclusão consecutiva de pacientes (inclusão em andamento), realizado em uma única instituição (Hospital do Coração - Associação do Sanatório Sírio - São Paulo, SP, Brasil). O estudo tem como objetivo investigar a evolução clínica tardia de pacientes tratados com stents farmacológicos. A partir de maio de 2002, a intervenção coronária percutânea com stents farmacológicos tem sido utilizada como estratégia preferencial nos pacientes encaminhados para tratamento percutâneo de rotina ou emergência na referida instituição. Foram in- of lesions in saphenous bypass grafts (RR 1.64, 95\% Cl, 1.14-2.35; $\mathrm{P}=0.008$ ). Conclusion: In the DESIRE registry, TLR in complex patients treated with DES was low and diabetes mellitus, treatment of lesions in saphenous bypass grafts and the use of Taxus stents were the only TLR predictors in the clinical follow-up of up to 8 years.

KEY-WORDS: Stents. Angioplasty. Coronary restenosis.

cluídos pacientes com pelo menos uma lesão $\geq 50 \%$ e anatomia favorável para intervenção coronária percutânea. Pelo protocolo, não existem limitações no que se refere ao número de lesões e/ou vasos que podem ser tratados ou ao número de stents farmacológicos implantados. O estudo está em consonância com a Declaração de Helsinque no que se refere à investigação em humanos, e foi aprovado pelo Comitê de Ética da referida instituição. Os resultados gerais do registro DESIRE já foram previamente publicados. ${ }^{5}$

\section{Procedimento}

As intervenções coronárias percutâneas foram realizadas de acordo com as diretrizes atuais e a estratégia final do procedimento foi deixada a critério do operador. Os seguintes stents farmacológicos de primeira e segunda gerações foram utilizados: Cypher $^{\circledR}$ (Cordis Corporation, Warren, Estados Unidos), Taxus ${ }^{\circledR}$ (Boston Scientific, Natick, Estados Unidos), Endeavor ${ }^{\circledR}$ (Medtronic, Santa Rosa, Estados Unidos), Xience $\vee^{\circledR}$ (Abbott Vascular, Abbott Park, Estados Unidos) e Biomatrix ${ }^{\circledR}$ (Biosensors International, Cingapura). A pré e a pós-dilatação com cateter-balão foi realizada a critério do operador. Ultrassonografia transluminal intracoronária para guiar o implante ótimo da endoprótese foi utilizada em casos selecionados. Também eram permitidos implante de múltiplos stents e/ou tratamento de múltiplas lesões (por meio de procedimento estagiado ou não). Em pacientes com síndrome coronária aguda, estudo angiográfico e intervenção coronária percutânea (quando indicada) foram realizados em $<48$ horas da hospitalização. O protocolo antitrombótico consistia na administração conjunta dos agentes antiplaquetários ácido acetilsalicílico e clopidogrel.

O pré-tratamento (ácido acetilsalicílico 300 mg e clopidogrel 300-600 mg) foi realizado 24 horas antes da intervenção, para casos eletivos, ou de 3 a 6 horas antes do procedimento, para a síndrome coronária aguda sem supradesnivelamento do segmento ST. No caso de intervenção coronária percutânea primária, a dose de ataque desses dois medicamentos foi administrada no momento do procedimento. Após a intervenção, a terapia com ácido acetilsalicílico era mantida indefinidamente, na dose de 100-200 mg/dia, e o clopidogrel, na dose de $75 \mathrm{mg}$ diários, era mantido inicialmente por 3 a 6 meses. A partir do final de 2006, seu uso foi estendido para 12 meses, de acordo com as novas 
recomendações do Food and Drug Administration (FDA). Durante o procedimento, foi administrada heparina endovenosa (70-100 unidades $/ \mathrm{kg}$ ) para manter o tempo de coagulação ativada $>250$ segundos (ou > 200 segundos no caso de ser administrado inibidor da glicoproteína IIb/IIla). Eletrocardiograma com 12 derivações foi obtido de rotina antes do procedimento, imediatamente após o procedimento e em 24 horas. Exames laboratoriais de rotina incluíram enzimas cardíacas creatina fosfoquinase $(\mathrm{CPK})$ e creatina quinase fração MB (CK-MB) massa - pré-procedimento ( $<24$ horas), 18-24 horas pós-procedimento, e diariamente até a alta hospitalar.

\section{Análise angiográfica}

A análise angiográfica qualitativa incluiu a avaliação do território miocárdico acometido, definido como a porção do músculo cardíaco irrigada por uma artéria epicárdica maior (descendente anterior, circunflexa e coronária direita) e seus respectivos ramos. Em relação à análise quantitativa, após a administração intracoronária de nitrato (50-200 $\mu$ g) foram obtidas a angiografia pré e pós-procedimento. As análises de angiografia coronária quantitativa foram realizadas por operadores experientes utilizando um sistema de análise quantitativa com detecção semiautomática das bordas luminais (Quantcor QCA-ACOM.PC versão 4.0 - Siemens, Munique, Alemanha). O diâmetro luminal mínimo e o diâmetro de referência do vaso, obtido pela média dos segmentos 5-10 mm proximal e distal à lesão-alvo, foram utilizados para calcular o diâmetro de estenose. Assim: diâmetro de estenose $=(1$ - diâmetro luminal mínimo/diâmetro de referência do vaso) x 100. Ganho imediato foi definido como a diferença do diâmetro luminal mínimo pré e pós-procedimento (diâmetro luminal mínimo pós-procedimento - diâmetro luminal mínimo pré-procedimento).

\section{Objetivos, definições e seguimento clínico}

O objetivo primário do estudo foi a ocorrência de eventos cardíacos adversos maiores no seguimento clínico tardio (> 6 meses). Os eventos cardíacos adversos maiores incluíram: morte cardíaca, infarto agudo do miocárdio e revascularização da lesão-alvo. Como regra, todas as mortes foram consideradas cardíacas, a não ser que uma causa não-cardíaca pudesse ser claramente estabelecida por estudo clínico e/ou patológico. O diagnóstico de infarto agudo do miocárdio foi baseado no aparecimento de nova onda-Q patológica em $\geq 2$ derivações contíguas no eletrocardiograma e/ou elevação da CK-MB massa > 3 vezes o limite superior da normalidade. A revascularização da lesãoalvo foi definida como nova intervenção coronária percutânea ou cirurgia de revascularização incluindo a lesão-alvo (compreendendo o segmento intrastent e os $5 \mathrm{~mm}$ proximais e distais às bordas da endoprótese) no seguimento tardio desses pacientes. A necessidade de novo procedimento de revascularização da lesão- alvo foi determinada pela presença de sintomas ou isquemia com prova funcional ou de acordo com a indicação do cardiologista de referência ou cardiologista intervencionista.

A trombose do stent foi definida de acordo com as proposições do Academic Research Consortium (ARC): definitiva (presença de síndrome coronária aguda com confirmação angiográfica ou anatomopatológica de oclusão do stent), provável (ocorrência de morte súbita $<30$ dias pós-procedimento índice ou infarto agudo do miocárdio no território miocárdico tratado, sem confirmação angiográfica da oclusão do stent) e possível (ocorrência de morte súbita > 30 dias após o procedimento índice). A trombose do stent ainda foi classificada de acordo com a ocorrência temporal: aguda ( $\leq 24$ horas do procedimento), subaguda (ocorrência entre 24 horas e 30 dias), tardia (ocorrência entre 1 mês e 12 meses pós-procedimento) e muito tardia (ocorrência > 12 meses pós-procedimento). O sucesso angiográfico foi definido como fluxo final TIMI 3 , ausência de dissecções e estenose residual $<20 \%$ pela angiografia coronária quantitativa. O seguimento clínico foi realizado 1 mês, 6 e 12 meses após o procedimento e anualmente até o oitavo ano de acompanhamento, e consistiu de visita médica agendada ou contato telefônico, realizado segundo protocolo prédefinido. Todos os eventos adversos reportados, incluindo trombose do stent, foram independentemente adjudicados por um Comitê de Eventos Clínicos, incluindo três experientes profissionais da área de cardiologia clínica e invasiva da instituição, em reunião específica para esse fim.

\section{Análise estatística}

As variáveis são apresentadas como frequências ou médias \pm desvio padrão. A ocorrência de nova revascularização da lesão-alvo em função do tempo foi descrita pela curva de Kaplan-Meier. Para identificar fatores associados ao desenvolvimento de revascularização da lesão-alvo foram utilizados modelos de riscos proporcionais de Cox. Todas as variáveis foram inicialmente analisadas em análises bivariadas e, posteriormente, de forma múltipla. A permanência das variáveis no modelo múltiplo foi baseada no teste de razão de verossimilhanças. Os resultados dos modelos de Cox foram apresentados em razões de risco e respectivos intervalos de confiança de 95\%. Valor de $\mathrm{P}<0,05$ foi considerado significativo. As análises foram realizadas com os programas estatísticos SPSS versão 16.0 (SPSS Inc., Chicago, Estados Unidos) e STATA versão 10 (StataCorp LP, College Station, Estados Unidos).

\section{RESULTADOS}

Entre maio de 2002 e fevereiro de 2010, um total de 3.320 pacientes foi incluído no registro. De maneira geral, os pacientes apresentaram perfil clínico de elevada complexidade (Tabela 1). Tal perfil complexo 
TABELA 1

Características clínicas basais

\begin{tabular}{lc}
\hline Variável & $\mathbf{n}=3.320$ \\
\hline Média das idades, anos & $64 \pm 11,3$ \\
Sexo masculino, \% & 76,9 \\
Hipertensão, \% & 76,6 \\
Diabetes melito, \% & 29,3 \\
Em uso de insulina & 5,6 \\
Dislipidemia, \% & 62,9 \\
Tabagismo atual, \% & 31,2 \\
História familiar de DAC, \% & 38,7 \\
IAM prévio, \% & 22,9 \\
ICP prévia, \% & 25,1 \\
CRM prévia, \% & 25,6 \\
AVC prévio, \% & 2,3 \\
Insuficiência renal, \% & 10,6 \\
Doença multiarterial, \% & 62 \\
Doença vascular periférica, \% & 3,8 \\
Apresentação clínica, \% & \\
Angina estável & 24,8 \\
Angina instável & 26,4 \\
IAM & 15,2 \\
Isquemia silenciosa & 33,6 \\
\hline Valores são expressos como frequência ou média \pm desvio \\
padrão. \\
AVC = acidente vascular cerebral; CRM cirurgia de re- \\
vascularização miocárdica; DAC arterial \\
coronária; IAM = infarto agudo do miocárdio; ICP = inter- \\
venção coronária percutânea; n = número de pacientes. \\
\hline
\end{tabular}

também se expressou nas indicações dos procedimentos, sendo $78,5 \%$ consideradas off-label.

Considerando os aspectos angiográficos, a artéria descendente anterior foi o vaso-alvo mais tratado (34,9\%); e considerando-se o total das lesões $(n=4.925), 20,1 \%$ delas eram localizadas na porção proximal desse vaso. A Tabela 2 apresenta os dados angiográficos.

\section{Dados do procedimento}

As lesões foram pré-dilatadas em $51,2 \%$ dos casos. A média de lesões e do número de vasos tratados por paciente foi de 1,48 $\pm 0,71$ e 1,37 $\pm 0,59$, respectivamente. No total, 5.320 stents farmacológicos foram implantados (média de stents por paciente: 1,60 $\pm 0,82$ stent) e $45 \%$ dos pacientes receberam múltiplos stents.

A média da pressão de liberação do stent foi de $14,4 \pm 2,9$ atm. A pós-dilatação com cateter-balão foi aplicada em 51,9\% dos casos, e as médias de extensão, diâmetro e pressão de insuflação do balão foram de $11,78 \pm 3,88 \mathrm{~mm}, 3,19 \pm 0,53 \mathrm{~mm}$, e 15,5 \pm 3,43 atm, respectivamente. Fluxo TIMI 3 pós-procedimento foi atingido em 99,7\% dos casos e o sucesso angiográfico foi de $99,4 \%$. Durante a fase hospitalar, todos os pa- cientes foram mantidos com terapia antiplaquetária dupla e a prescrição foi mantida na alta hospitalar de acordo com o protocolo. A Tabela 3 apresenta os resultados da angiografia coronária quantitativa.

\section{Seguimento angiográfico}

Nesse Registro, o reestudo angiográfico não é obrigatório pelo protocolo. Dos 3.320 pacientes, 687 (20,7\% do total) realizaram reestudo angiográfico em nossa instituição. Em 77 pacientes (11,2\% dos pacientes submetidos a reestudo e $2,32 \%$ do total de pacientes) foi detectada reestenose angiográfica (definida como presença de nova estenose $>50 \%$ no segmento intrastent ou nos $5 \mathrm{~mm}$ proximais ou distais à endoprótese). A indicação de nova cinecoronariografia no seguimento dos pacientes foi determinada por recorrência dos sintomas e/ou nova prova funcional positiva para isquemia em $51 \%$ dos pacientes ou por decisão do clínico de referência para seguimento dos pacientes. No reestudo angiográfico (tempo médio: 12 meses), a média de perda luminal tardia foi de 0,48 $\pm 0,28 \mathrm{~mm}$, e $11,2 \%$ apresentaram reestenose $(85,3 \%$ padrão focal, tipo IB/IC pela classificação de Mehran). Também, o uso de stents farmacológicos Cypher foi menor no grupo com reestenose, comparativamente ao grupo sem reestenose $(67 \%$ vs. $86 \% ; P<0,001)$.

\section{Eventos clínicos}

$\mathrm{Na}$ fase intra-hospitalar, morte cardíaca ocorreu em $0,6 \%$ dos pacientes e $0,33 \%$ evoluíram com infarto agudo do miocárdio. O seguimento clínico tardio até oito anos (mediana 3,4 anos) foi alcançado em 98\% dos pacientes (por consulta médica agendada ou contato telefônico). As taxas cumulativas de eventos cardíacos adversos maiores foram de 10,88\%, incluindo óbito cardíaco em 2,5\%, infarto agudo do miocárdio em $4,5 \%$, e revascularização da lesão-alvo em 3,88\% dos pacientes. Durante o período de seguimento, 52 (1,6\%) pacientes evoluíram com trombose do stent. A maioria das tromboses (65\%) foi classificada como definitiva, com confirmação angiográfica $(n=34)$. Quando consideradas apenas as tromboses do stent definitivas, a média de tempo até o evento foi de 1,8 ano.

\section{Preditores de eventos adversos até oito anos de seguimento clínico}

Os preditores independentes de eventos cardíacos adversos maiores foram: indicação off-label [razão de risco (RR) 1,77, intervalo de confiança de 95\% (IC 95\%) 1,31-2,29; $P<0,001$ ], doença multiarterial (RR 1,31, IC 95\% 1,04-1,66; $P=0,02)$, lesão-alvo em ponte de safena (RR 1,63, IC 95\% 1,22-2,18; P = 0,001), doença multiarterial (RR 1,39, IC 95\% 1,03-1,87; $\mathrm{P}<0,001$ ), estenose residual intrastent (RR 1,3 - por aumento de unidade \%, IC 95\% 1,10-1,50; P = 0,034), diabetes melito (RR 1,24, IC 95\% 1,01-1,51; P = 0,042) e insuficiência renal (RR 1,44, IC 95\% 1,10-1,89; $P=0,008)$. A Figura 1 mostra a curva de sobrevida livre de 
TABELA 2

Características angiográficas

\begin{tabular}{lc}
\hline Variável & $\mathbf{n}=3.320$ \\
\hline Território miocárdico acometido (número de territórios = 4.232), \% & 42,3 \\
DA & 28,8 \\
Cx & 27,5 \\
CD & 1,4 \\
TCE & 5,8 \\
Características da lesão (número de lesões = 4.925), \% & 4,8 \\
Ponte de veia de safena & 3,6 \\
Localização ostial & 5 \\
Bifurcação & 24,2 \\
Angulação > 45 graus & 25,7 \\
Excentricidade & 4,2 \\
Calcificação moderada ou grave & 68,4 \\
Reestenose intrastent & 12,1 \\
Lesão tipo B2 ou C & \\
Fração de ejeção do VE <40\% & \\
\hline
\end{tabular}

$\mathrm{CD}=$ artéria coronária direita; $\mathrm{Cx}=$ artéria circunflexa; $\mathrm{DA}=$ artéria descendente anterior; $\mathrm{n}=$ número de pacientes; $\mathrm{TCE}=$ tronco de coronária esquerda; VE = ventrículo esquerdo.

TABELA 3

Angiografia coronária quantitativa (número de lesões $=4.925$ )

\begin{tabular}{lcr}
\hline Variável quantitativa & Pré-procedimento & Pós-procedimento \\
\hline Extensão da lesão, mm & $16,93 \pm 8,49$ & - \\
DRV, mm & $2,77 \pm 0,48$ & - \\
DLM, mm & $0,91 \pm 1,07$ & $2,75 \pm 0,82$ \\
DE, mm & $67,27 \pm 11,2$ & $4,37 \pm 3,98$ \\
Ganho imediato, mm & - & $1,85 \pm 0,55$ \\
\hline
\end{tabular}

Valores são expressos como média \pm desvio padrão.

Análise do segmento intrastent.

$\mathrm{DE}=$ diâmetro de estenose; DLM = diâmetro luminal mínimo; DRV = diâmetro de referência do vaso.

revascularização da lesão-alvo na população geral. A Tabela 4 apresenta a análise univariada para identificação de preditores de revascularização da lesão-alvo e os preditores independentes de revascularização da lesão-alvo identificados no modelo multivariado são apresentados na Tabela 5. Na análise univariada os seguintes preditores de revascularização da lesão-alvo foram identificados: diabetes melito (RR 1,88, IC 95\% $1,08-3,27 ; \mathrm{P}=0,025)$, uso de stents farmacológicos Taxus (RR 1,73, IC 95\% 1,20-2,50; P = 0,003), lesão ostial (RR 1,82, IC 95\% 1,1-2,98; $\mathrm{P}=0$ 0,018), ponte de safena (RR 2,36, IC 95\% 1,55-3,50; $P=0,0001$ ), lesões reestenóticas ( $R R 1,95$, IC 95\% 1,17-3,24; $\mathrm{P}=0$ 0,009). Após análise multivariada, permaneceram como preditores independentes: diabetes melito (RR 1,94, IC 95\% $1,35-2,78 ; P \leq 0,001)$, uso de stents farmacológicos
Taxus (RR 1,89, IC 95\% 1,40-2,53; $\mathrm{P} \leq 0,001)$ e tratamento de lesões em pontes de safena (RR 1,64, IC 95\% 1,14-2,35; $P=0,008$ ).

\section{DISCUSSÃO}

A presente análise revela que a eficácia do uso de stents farmacológicos em populações do mundo real é similar aos achados dos estudos randomizados. Assim, demonstram-se no seguimento tardio (até 8 anos com mediana de 3,4 anos) taxas relativamente baixas de reestenose angiográfica $(11,2 \%)$ e nova revascularização da lesão-alvo $(3,88 \%)$.

Deve-se lembrar que nem todos os pacientes que necessitaram de nova revascularização da lesão-alvo no seguimento clínico desse Registro foram submetidos, 
em nossa instituição, ao reestudo angiográfico e ao novo procedimento de revascularização. O que justifica a diferença nos porcentuais de reestenose binária quando se considera a população do reestudo $(n=687 / 77$ pacientes/11,2\%) vs. a população total do Registro ( $n=3.320 / 77$ pacientes/2,32\%). Isso ressalta a importância do seguimento clínico dos pacientes de uma grande instituição por meio de visita médica agendada ou contato telefônico, com o propósito de aferir adequadamente a incidência dos eventos clínicos.

Essas diferenças entre as incidências de reestenose angiográfica e reestenose clínica são conhecidas desde os estudos de angioplastia com balão e se repeti-

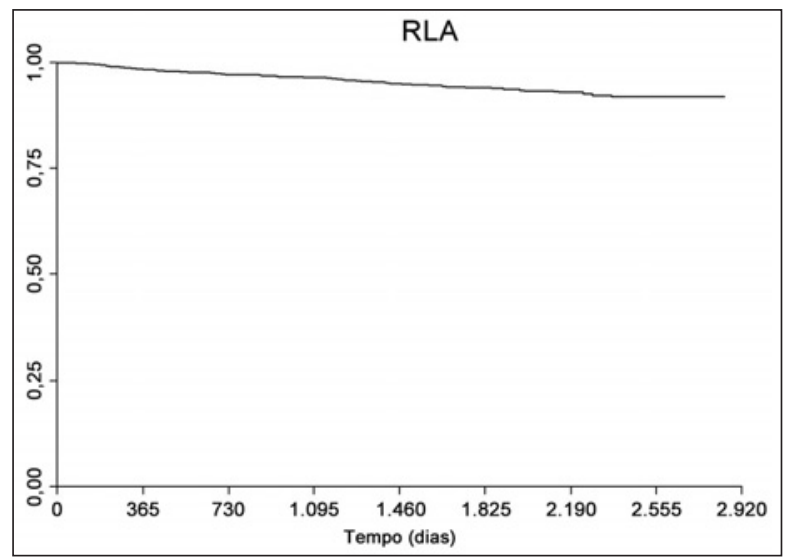

Figura 1 - Curva de sobrevivência livre de revascularização da lesão-alvo (RLA) do Registro DESIRE. ram com os stents não-farmacológicos. ${ }^{6}$ A recorrência de sintomas e/ou prova funcional positiva para isquemia foi responsável pela indicação de reestudo em 51\% dos casos, e a outra metade dos pacientes submetida ao reestudo angiográfico era assintomática e/ou sem evidência objetiva de isquemia miocárdica. Assim, acreditamos que o reestudo angiográfico não protocolar na prática clínica diária não parece influenciar de forma exagerada a indicação de nova revascularização, mantendo níveis aceitáveis de nova revascularização da lesão-alvo, desde que se valorize a evidência subjetiva e/ou objetiva de isquemia. ${ }^{7}$

$\mathrm{O}$ achado de perda luminal tardia de 0,48 $\pm 0,28 \mathrm{~mm}$ demonstra não haver efeito de classe entre os stents farmacológicos quando se refere à inibição da hiperplasia neointimal. Os diferentes dispositivos usados na população deste registro apresentam poder antiproliferativo distinto, que se relaciona ao fármaco, às distintas plataformas metálicas e aos polímeros. Adicionalmente, o uso do stent Cypher foi menor no grupo que apresentou reestenose angiográfica, em comparação aos que não evoluíram com reestenose, e talvez reflita sua superioridade em situações de maior complexidade angiográfica, como os vasos de menor calibre., ${ }^{8,9}$

No Registro DESIRE, o uso de stents farmacológicos Taxus foi preditor independente da necessidade de novo procedimento na lesão-alvo no seguimento tardio dessa coorte. Esse achado está de acordo com outras experiências anteriormente publicadas em relação aos pacientes consecutivos prospectivamente acompanhados em grandes coortes. ${ }^{10-13}$

TABELA 4

Análise univariada para preditores de revascularização da lesão-alvo

\begin{tabular}{lccc}
\hline Variável & Razão de risco & IC 95\% & Valor de P \\
\hline Diabetes melito & 1,88 & $1,08-3,27$ & 0,025 \\
Uso de stent Taxus & 1,73 & $1,20-2,50$ & 0,007 \\
Lesão ostial & 1,82 & $1,10-2,98$ & 0,018 \\
Ponte de safena & 2,36 & $1,55-3,50$ & 0,001 \\
Lesão reestenótica & 1,95 & $1,17-3,24$ & 0,009 \\
\hline
\end{tabular}

IC = intervalo de confiança.

TABELA 5

Análise multivariada demonstrando preditores independentes significativos de revascularização da lesão-alvo

\begin{tabular}{lccc}
\hline Variável & Razão de risco & IC 95\% & Valor de P \\
\hline Diabetes melito & 1,94 & $1,35-2,78$ & $<0,001$ \\
Uso de stent Taxus & 1,89 & $1,35-2,53$ & $<0,001$ \\
Ponte de safena & 1,64 & $1,14-2,35$ & 0,008 \\
\hline
\end{tabular}

$\mathrm{IC}=$ intervalo de confiança. 
O presente estudo também identificou o diabetes melito como preditor de revascularização da lesãoalvo. Ainda há achados controversos sobre a associação dessa característica clínica com o evento que representa a falência do uso do stent farmacológico. ${ }^{11-13}$ Entretanto, sua relação com desfechos combinados de eventos cardíacos adversos maiores já tenha sido descrita por pesquisadores do Registro DESIRE. ${ }^{14}$ Embora o diabetes melito se associe claramente à reestenose angiográfica, não há dados consistentes relacionando essa variável à revascularização da lesão-alvo. Isso ressalta a distinção entre o achado angiográfico de reestenose intrastent e a necessidade de novo procedimento guiado por sintomas e/ou isquemia miocárdica. $\mathrm{Na}$ experiência de Maeng et al. ${ }^{13}$, que avaliaram o registro dinamarquês com 4.432 pacientes, o diabetes melito não foi considerado preditor de revascularização da lesão-alvo; mas em publicação recente, Takasawa et al. ${ }^{15}$ referem-se ao diabetes melito como preditor independente de nova revascularização após o tratamento de reestenose de stents farmacológicos. O registro italiano REAL (com 10.629 pacientes, sendo 3.064 submetidos a implante de stents farmacológicos), outro grande registro, demonstrou o diabetes melito como preditor independente de revascularização da lesão-alvo. ${ }^{16}$

Embora a extensão da lesão tenha sido associada como preditor independente de revascularização da lesão-alvo na era dos stents não-farmacológicos, tal fato permanece controverso quando se consideram os dispositivos com eluição de medicamentos. Kastrati et al. ${ }^{11}$, Maeng et al. ${ }^{13}$ e Marzocchi et al. ${ }^{16}$ também não encontraram associação entre extensão da lesão e revascularização da lesão-alvo. Esse último pesquisador, no já citado registro italiano multicêntrico, demonstrou que, mesmo com a extensão média da lesão de 20,4 \pm 6 mm, a extensão da lesão não foi preditora de revascularização da lesão-alvo. Por outro lado, Lee et al. ${ }^{12}$, ao investigar os preditores de reestenose angiográfica numa população de 1.795 pacientes submetidos a implante de stents farmacológicos, encontraram a extensão da lesão > $20 \mathrm{~mm}$ como preditora de desfecho angiográfico. Ressalte-se que esse pesquisador avaliou especificamente reestenose e não revascularização da lesão-alvo. No Registro DESIRE, os valores médios da extensão da lesão são menores que $20 \mathrm{~mm}$ $(16,78 \pm 7,48 \mathrm{~mm}$ e $16,93 \pm 8,53 \mathrm{~mm}$, respectivamente, para os grupos com e sem revascularização da lesão-alvo).

Também existem dados conflitantes na literatura em relação ao diâmetro de referência como preditor de revascularização da lesão-alvo. No Registro DESIRE, assim como na experiência de Lee et al. ${ }^{12}$, o diâmetro de referência não foi preditor de revascularização da lesão-alvo; contudo, outros autores demonstraram o diâmetro de referência como preditor independente de revascularização da lesão-alvo mesmo na era dos stents farmacológicos. ${ }^{11,13,16}$ É válido ponderar que Kastrati et al. ${ }^{11}$, em sua interessante aplicação do chamado modelo CART (classification and regression tree), encontraram valor preditivo independente no diâmetro de referência, entretanto com impacto preponderante em vasos com diâmetro de referência $<2,6 \mathrm{~mm}$. No Registro DESIRE, contudo, o diâmetro de referência é, em média, mais elevado $(2,75 \pm 0,45 \mathrm{~mm}$ e 2,78 \pm $0,49 \mathrm{~mm}$, respectivamente, nos grupos com e sem revascularização da lesão-alvo).

Por último, o achado de intervenção em pontes de safena como preditor independente de revascularização da lesão-alvo está de acordo com publicações prévias do DESIRE, quando apresentados seus resultados em seguimento clínico menos extenso. ${ }^{17} \mathrm{O}$ impacto negativo dessa variável na prevalência de eventos cardíacos adversos maiores já está bem demonstrado na literatura. ${ }^{18,19}$

\section{Limitações do estudo}

Este estudo representa a experiência clínica de uma instituição em que stents farmacológicos têm sido utilizados como estratégia preferencial em pacientes com indicação de intervenção coronária percutânea. Como o Registro DESIRE conta com inclusão contínua de pacientes por tempo indeterminado, a análise atual reflete os achados referentes a um período preestabelecido (maio de 2002 a fevereiro de 2010). No período do estudo, foram excluídos os pacientes tratados com stents não-farmacológicos ou com a combinação de stents farmacológicos e stents não-farmacológicos. Por último, os diferentes tipos de stents farmacológicos foram implantados em proporções não-balanceadas, tendo sido as indicações baseadas em disponibilidade, anatomia e localização da lesão, quadro clínico, e preferência do operador.

\section{CONCLUSÕES}

No Registro DESIRE, a revascularização da lesãoalvo em pacientes complexos tratados com stents farmacológicos foi baixa, e o diabetes melito, a intervenção em pontes de safena e o uso do stent Taxus foram os únicos preditores independentes desse evento em um acompanhamento clínico de até oito anos.

\section{AGRADECIMENTOS}

Os autores reconhecem a dedicação, competência e significativa colaboração dos seguintes profissionais envolvidos no Registro DESIRE: Cantídio Campos, Mariana T. Carballo, Valéria Ranieri e Wagner Pinto.

\section{CONFLITO DE INTERESSES}

Os autores declararam inexistência de conflito de interesses relacionado a este manuscrito.

\section{REFERÊNCIAS}

1. Morice MC, Serruys PW, Sousa JE, Fajadet J, Ban Hayashi $\mathrm{E}$, Perin $\mathrm{M}$, et al. A randomized comparison of a sirolimus- 
eluting stent with a standard stent for coronary revascularization. RAVEL Study Group. N Engl J Med. 2002;346(23): 1773-80.

2. Moses JW, Leon MB, Popma JJ, Fitzgerald PJ, Holmes DR, $\mathrm{O}^{\prime}$ Shaughnessy C, et al. Sirolimus-eluting stents versus standard stents in patients with stenosis in a native coronary artery. SIRIUS Investigators. N Engl J Med. 2003;349(14):1315-23.

3. Stone GW, Ellis SG, Cox DA, Hermiller J, O'Shaughnessy C, Mann JT, et al. A polymer-based, paclitaxel-eluting stent in patients with coronary artery disease. TAXUS-IV Investigators. N Engl J Med. 2004;350(3):221-31.

4. Sousa JE, Costa MA, Tuzcu EM, Yadav JS, Ellis S. New frontiers in interventional cardiology. Circulation. 2005;111(5):671-81.

5. Sousa AGMR, Costa Jr JR, Moreira A, Costa RA, Cano MN, Andrade GAM, et al. Evolução clínica tardia dos stents farmacológicos: segurança e eficácia até cinco anos do registro DESIRE. Rev Bras Cardiol Invasiva. 2007;15(3):221-7.

6. Kastrati A, Schomig A, Elezi S, Schühlen H, Dirschinger J, Hadamitzky $\mathrm{M}$, et al. Predictive factors of restenosis after coronary stent placement. J Am Coll Cardiol. 1997;30(6):1428-36.

7. Uchida T, Popma J, Stone GW, Ellis SG, Turco MA, Ormiston JA, et al. The clinical impact of routine angiographic followup in randomized trials of drug-eluting stents: a critical assessment of "oculostenotic" reintervention in patients with intermediate lesions. JACC Cardiovasc Interv. 2010;3(4):403-11.

8. Mauri L, Orav EJ, O'Malley AJ, Moses JW, Leon MB, Holmes $D R$, et al. Relationship of late loss in lumen diameter to coronary restenosis in sirolimus-eluting stents. Circulation. 2005; 111(3):321-7.

9. Lemos PA, Hoye A, Goedhart D, Arampatzis CA, Saia F, van der Giessen WJ, et al. Clinical, angiographic, and procedural predictors of angiographic restenosis after sirolimuseluting stent implantation in complex patients: an evaluation from the Rapamycin-Eluting Stent Evaluated at Rotterdam Cardiology Hospital (RESEARCH) study. Circulation. 2004; 109(11):1366-70.

10. Kastrati A, Dibra A, Eberle S, Mehilli J, Suárez de Lezo J, Goy J, et al. Sirolimus-eluting stents vs paclitaxel-eluting stents in patients with coronary artery disease meta-analysis of randomized trials. JAMA. 2005;294(5):819-25.
11. Kastrati A, Dibra A, Mehilli J, Mayer S, Pinieck S, Pache J, et al. Predictive factors of restenosis after coronary implantation of sirolimus- or paclitaxel-eluting stents. Circulation. 2006; 113(19):2293-300.

12. Lee CW, Park DW, Lee BK, Kim YH, Hong MK, Kim JJ, et al. Predictors of restenosis after placement of drug-eluting stents in one or more coronary arteries. Am J Cardiol. 2006; 97(4):506-11.

13. Maeng M, Okkels Jensen L, Rasmussen K, Flensted Lassen J, Romer Krusell L, Thayssen P, et al. Target lesion revascularisation in patients treated with a sirolimus-eluting or paclitaxel-eluting stent. Heart. 2007;93(6):694-7.

14. Moreira A, Sousa AGMR, Costa Jr JR, Costa RA, Maldonado $\mathrm{GA}$, Cano $\mathrm{MN}$, et al. Evolução tardia após intervenção coronária percutânea com stents farmacológicos em pacientes diabéticos do Registro DESIRE. Rev Bras Cardiol Invasiva. 2008;16(2):185-92.

15. Takasawa Y, Lijima R, Shiba M, Nakamura M, Sugi K. Predictor of subsequent target lesion revascularization in patients with drug-eluting stent restenosis undergoing percutaneous coronary intervention. J Cardiol. 2010;55(3):391-6.

16. Marzocchi A, Saia F, Piovaccari G, Manari A, Aurier E, Benassi A, et al. Long-term safety and efficacy of drug-eluting stents: two-year results of the REAL (REgistro AngiopLastiche dell'Emilia Romagna) multicenter registry. Circulation. 2007;115(25):3181-8.

17. Sousa AGMR, Costa Jr JR, Moreira A, Cano MN, Maldonado GA, Costa RA, et al. Long-term clinical outcomes of the DrugEluting Stents in the Real World (DESIRE) registry. J Interv Cardiol. 2008;21(4):307-14.

18. Costa Jr JR, Sousa AGMR, Moreira A, Costa RA, Maldonado $\mathrm{GA}$, Cano $\mathrm{MN}$, et al. Intervenção coronária percutânea em pontes de veia de safena com uso de stents farmacológicos: resultados agudos e tardios dos pacientes incluídos no Registro DESIRE. Rev Bras Cardiol Invasiva. 2009;17(2): 202-8.

19. Van Twisk PH, Daemen J, Serruys PW, van Domburg RT, Serruys PW. Four-year safety and efficacy of the unrestricted use of sirolimus- and paclitaxel-eluting stents in coronary artery bypass grafts. Eurointervention. 2008;4(3):311-7. 